
И МОЛОДОГО ВОЗРАСТА С ГИПОТАЛАМИЧЕСКИМ СИНДРОМОМ

\author{
(с Л.К. Церцвадзе' , М.В. Авдеева ${ }^{1,2 *}$, Л.В. Щеглова', В.С. Василенко ${ }^{1}$
}

${ }^{1}$ Санкт-Петербургский государственный педиатрический медицинский университет, Санкт-Петербург, Россия ${ }^{2}$ Северо-Западный государственный медицинский университет им. И.И. Мечникова, Санкт-Петербург, Россия

Обоснование. Эндотелиальная дисфункция является ранней, но обратимой стадией атеросклероза. Изменение функционального состояния сосудистого эндотелия растущего организма может являться основой развития многих заболеваний в зрелом возрасте. Изучение структурно-функционального состояния периферических артерий у юношей с гипоталамическим синдромом крайне важно для лучшего понимания механизмов формирования кардиометаболических рисков в молодом возрасте.

Цель. Сравнить структурно-функциональное состояние сосудистой стенки у юношей с гипоталамическим синдромом и конституционально-экзогенным ожирением.

Memoды. Обследовано 360 лиц мужского пола (средний возраст 21,27士2,44 года), которые разделены на 3 группы: 1 группа - с гипоталамическим синдромом ( $\mathrm{n}=242) ; 2$ группа - с конституционально-экзогенным ожирением ( $\mathrm{n}=98)$; контроль - практически здоровые лица $(\mathrm{n}=20)$. Диагностическим критерием гипоталамического синдрома считалось ожирение с розовыми стриями. Проводился межгрупповой сравнительный анализ результатов клинического, лабораторного и инструментального обследования.

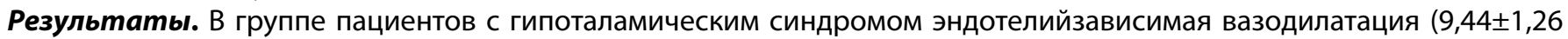

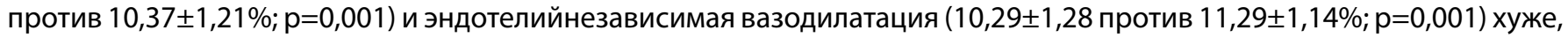
чем при конституционально-экзогенном ожирении, а показатель эндотелийзависимой вазодилатации ниже общепринятой нормы. Помимо этого, у пациентов с гипоталамическим синдромом жесткость сосудистой стенки выше, чем

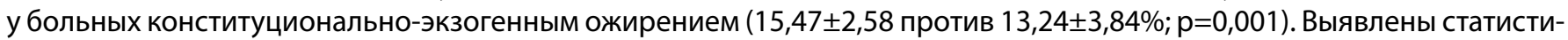
чески значимые корреляции между структурно-функциональным состоянием периферических артерий и гемодинамическими, гормональными, метаболическими изменениями, уровнем С-реактивного белка $(p<0,05)$.

Заключение. Полученные данные свидетельствуют об ухудшении функции эндотелия и повышении жесткости сосудистой стенки у больных, страдающих ожирением, независимо от его этиологии. У пациентов с гипоталамическим синдромом отмечаются более выраженные структурно-функциональные изменения сосудистой стенки, чем при конституционально-экзогенном ожирении.

КЛЮЧЕВЫЕ СЛОВА: гипоталамический синдром; ожирение с розовыми стриями; эндотелиальная дисфункция.

\title{
MARKERS OF ENDOTHELIAL DYSFUNCTION IN ADOLESCENT AND YOUNG PATIENTS WITH HYPOTHALAMIC SYNDROME
}

\author{
(c) Lana K. Tsertsvadze1, Marina V. Avdeeva ${ }^{1,2 *}$, Larisa V. Scheglova', Vladimir S. Vasilenko'
}

${ }^{1}$ St. Petersburg State Pediatric Medical University, St. Petersburg, Russia

${ }^{2}$ North-Western State Medical University named after I.I. Mechnikov, St. Petersburg, Russia

BACKGROUND: Endothelial dysfunction is the first but reversible stage of atherosclerosis. A change in the functional state of the vascular endothelium, especially of a growing organism, can be the basis for the development of many diseases in adulthood. The study of the structural and functional state of peripheral vessels of adolescents with hypothalamic syndrome is extremely important for understanding of the mechanisms of formation of cardiometabolic risks.

AIMS: to compare the structural and functional state of the vascular wall of young men with hypothalamic syndrome and constitutionally exogenous obesity.

METHODS: During the study 360 males were examined (average age 21.27 \pm 2.44 years) and divided into 3 groups: group 1 with hypothalamic syndrome $(n=242)$; group 2 - with constitutionally exogenous obesity $(n=98)$; control group - practically healthy individuals $(n=20)$. Hypothalamic syndrome was verified in the presence of a symptom complex, including obesity and pink striae. Cross-group comparative analysis of results of clinical, laboratory and instrumental examination was carried out.

RESULTS: In the group of patients with hypothalamic syndrome, endothelium-dependent vasodilatation ( $9.44 \pm 1.26$ versus $10.37 \pm 1.21 \% ; p=0.001)$ and endothelium-independent vasodilatation $(10.29 \pm 1.28$ versus $11.29 \pm 1.14 \% ; p=0.001)$ is worse than in the group of patients with constitutionally exogenous obesity, and the rate of endothelium-dependent vasodilatation is lower than the generally accepted norm. In addition, among patients with hypothalamic syndrome, the stiffness of the vascular wall is higher than among patients with constitutionally exogenous obesity $(15.47 \pm 2.58$ versus $13.24 \pm 3.84 \%$; $p=0.001)$. Statistically significant correlations were revealed between the structural and functional state of peripheral arteries and hemodynamic, hormonal, metabolic changes, and the level of C-reactive protein $(p<0.05)$.

*Автор, ответственный за переписку / Corresponding author. 
CONCLUSIONS: The obtained data indicate a deterioration of endothelial function and an increase in vascular wall stiffness in obese patients, regardless of its etiology. The patients with hypothalamic syndrome have more pronounced structural and functional changes in the vascular wall are observed than patients with constitutionally exogenous obesity.

KEYWORDS: hypothalamic syndrome; obesity with pink striae; endothelial dysfunction.

\section{ОБОСНОВАНИЕ}

За последние 30 лет распространенность ожирения в мире удвоилась [1], а проблема ожирения у детей и подростков стала одной из наиболее важных проблем здравоохранения многих развитых стран [2]. В Санкт-Петербурге, как и в других регионах России, также отмечается рост первичной и общей заболеваемости ожирением среди детей и подростков [3, 4]. Детское и подростковое ожирение впоследствии становится истоком развития большинства эндокринных и сердечно-сосудистых заболеваний в зрелом возрасте $[5,6,7]$. Ожирение связано с инсулинорезистентностью, периферической вазоконстрикцией, повышением активности ренин-ангиотензин-альдостероновой и симпатоадреналовой систем, воспалением жировой ткани, нарушением секреторной активности адипоцитов, вследствие чего повышается секреция провоспалительных цитокинов, свободных жирных кислот, активных форм кислорода, других метаболитов и развивается хроническое медленно прогрессирующее воспаление, оксидативный стресс и дисфункция эндотелия [8, 9]. Жировая ткань может продуцировать соединения, способные ухудшать функцию эндотелия, среди которых лептин, резистин, фактор некроза опухоли-альфа, интерлейкин-6, белок-хемоаттрактант моноцитов-1, ингибитор активатора плазминогена-1, адипонектин и белки ренин-ангиотензиновой системы [10].

Ожирение с розовыми стриями является ведущим симптомом гипоталамического синдрома пубертатного периода $[11,12]$. Данная эндокринная патология является следствием первичной незрелости гипоталамических структур [13] или вторичной дисфункции, вызванной структурно-функциональным повреждением гипоталамуса $[14,15]$. Проявления гипоталамического синдрома в виде розовых стрий, высокорослости, вегетативной дисфункции могут встречаться у 46-80\% детей и подростков, страдающих ожирением $[16,17]$.

За последние 20 лет распространенность гипоталамического синдрома пубертатного периода выросла в 2 раза [18], что объясняет актуальность данной проблемы, а также интерес для научных исследований. Вместе с тем гипоталамическое ожирение изучено недостаточно, о чем свидетельствует малое количество исследований и публикаций на эту тему [14]. Следует отметить, что при гипоталамическом синдроме пубертатного периода исследовано ограниченное количество маркёров кардиометаболического риска [19]. При этом роль эндотелиальной дисфункции, как маркера кардиометаболического риска у больных гипоталамическим синдромом неясна. Помимо этого неизвестна роль гормонально-метаболических нарушений, развивающихся при гипоталамическом синдроме, в генезе эндотелиальной дисфункции. В связи с этим актуальность исследования определяется необходимостью изучения структурно-функционального состояния сосудистой стенки у пациентов с гипоталамическим синдромом и его связи с развивающимися при этом гормонально-метаболическими нарушениями.

\section{ЦЕЛЬ}

Сравнить структурно-функциональное состояние сосудистой стенки у юношей с гипоталамическим синдромом и конституционально-экзогенным ожирением.

\section{МЕТОДЫ}

Дизайн исследования

Дизайн исследования предтавлен на рис. 1. Обсервационное одноцентровое одномоментное выборочное контролируемое нерандомизированное исследование.

\begin{tabular}{|c|c|c|}
\hline & \multirow{2}{*}{$\begin{array}{c}360 \text { лиц мужского пола } 18-27 \text { лет } \\
\text { (средний возраст } 21,2 \pm 2,4 \text { года) } \\
\downarrow\end{array}$} & \multirow[b]{2}{*}{$\downarrow$} \\
\hline$\downarrow$ & & \\
\hline 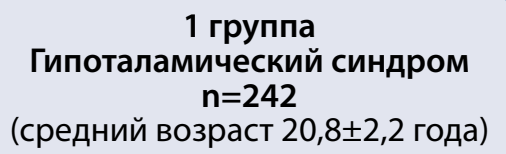 & 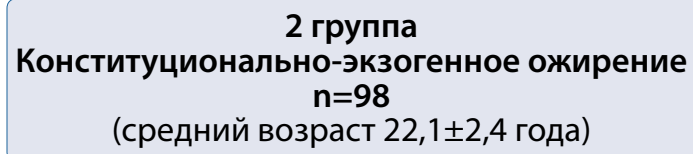 & $\begin{array}{c}\text { Контрольная группа } \\
\mathbf{n}=20 \\
\text { (средний возраст } 21,9 \pm 3,3 \text { года) }\end{array}$ \\
\hline$\downarrow$ & $\downarrow$ & $\downarrow$ \\
\hline $\begin{array}{ll} & \\
\text { - } & \text { Клинико-анамнестическое и } \\
\text { - } & \text { Объективный осмотр на нал } \\
\text { - } & \text { Исследование трофологиче } \\
& \text { инсулин, индекс НОМА, инд } \\
\text { - } \quad \text { Биохимические исследован } \\
\quad \text { (общий холестерин, глюкоза } \\
\text { - Липидный спектр крови } \\
\text { - } \quad \text { С-реактивный белок } \\
\text { - Исследование функциональ } \\
\text { - Определение эндотелийзав } \\
\text { - Определение возраста сосу, }\end{array}$ & $\begin{array}{l}\text { Обследование: } \\
\text { следование (дебют ожирения) } \\
\text { 1чие розовых стрий, гинекомастии } \\
\text { кого статуса (ИМТ, объем талии, объем бедер } \\
\text { ормонального статуса (общий тестостерон, ко } \\
\text { кс анаболизма, ФСГ, ЛГ, эстрадиол, ТТГ, ТЗ, Т4, с } \\
\text { я } \\
\text { гликированный гемоглобин, АСТ, АЛТ, креати } \\
\text { ого состояния сердца (суточное мониториров } \\
\text { симой и эндотелийнезависимой вазодилатаци } \\
\text { ов, жёсткости сосудов, индекса стресса }\end{array}$ & $\begin{array}{l}\text { др.) } \\
\text { изол, пролактин, базальный } \\
\text { титела к тиреопероксидазе) } \\
\text { нн, мочевина, и др.) } \\
\text { ние АД, эхокардиография) }\end{array}$ \\
\hline
\end{tabular}

Рисунок 1. Дизайн исследования 
Критерии соответствия

Критерии включения: мужской пол; европеоидная раса; призывной возраст 18-27 лет (поздний пубертат или молодой возраст), добровольное информированное согласие на участие в исследовании.

Другие критерии включения/невключения не применялись.

\section{Условия проведения}

Исследование выполнено на базе эндокринологического отделения СПб ГБУЗ «Городская Мариинская больница».

\section{Продолжительность исследования}

Продолжительность набора участников в исследование в соответствии с планом составила 3 года. Исследование проводилось в период с 2017 по 2019 гг.

\section{Описание медицинского вмешательства}

Всех участников обследовали по единому протоколу:

- общий осмотр для выявления гинекомастии, розовых стрий, определения росто-весовых показателей, объема талии, объема бедер;

- $\quad$ забор венозной крови натощак для определения сывороточных уровней общего тестостерона, кортизола, пролактина, базального инсулина, фолликулостимулирующего гормона, лютеинизирующего гормона, эстрадиола, общего холестерина, глюкозы, гликированного гемоглобина, АСТ, АЛТ, липидного спектра крови, С-реактивного белка;

- для оценки функционального состояния кардиоваскулярной системы проводилось суточное мониторирование артериального давления (АД) и эхокардиографическое исследование;

- для оценки функции эндотелия сосудов проводились исследования методами плетизмографии и ультразвукового сканирования;

\section{Основной исход исследования}

Основными оцениваемыми параметрами в рамках исследования явились показатели эндотелийзависимой вазодилатации (ЭЗВД, потокзависимая вазодилатация); эндотелийнезависимой вазодилатации (ЭНЗВД); показатель региональной жесткости артериального русла - индекс аугментации, нормализованный до чСС=75 в минуту (Alp75, augmentation index); ангиологический возраст (определяется на основании возрастного индекса, рассчитанного с использованием математических моделей).

\section{Дополнительные исходы исследования}

Дополнительные оцениваемые параметры: показатели суточного мониторирования АД (ЧСС; среднесуточные показатели АД; вариабельность АД); эхокардиографические показатели (относительная толщина стенки левого желудочка; масса миокарда левого желудочка, толщина межжелудочковой перегородки, толщина задней стенки левого желудочка); индекс массы тела (ИМТ); объем талии; объем бедер; соотношение объем талии/объем бедер; уровни С-реактивного белка, фолликулостимулирующего гормона, лютеинизирующего гормона, пролактина, кортизола, инсулина, индекс НОМА; липидный спектр крови.

\section{Анализ в подгруппах}

Участников исследования разделили на три когорты: с гипоталамическим синдромом; с конституционально-экзогенным ожирением; практически здоровых лиц.

Гипоталамический синдром (гипоталамическое ожирение, ожирение с розовыми стриями) верифицировался при наличии симптомокомплекса, включающего ожирение и розовые стрии [12].

Группу с гипоталамическим синдромом составили лица мужского пола; призывного возраста с признаками гипоталамического синдрома; без сахарного диабета; без аденомы гипофиза; без синдрома Иценко-Кушинга.

Группу с конституционально-экзогенным ожирением составили лица мужского пола; призывного возраста с признаками абдоминального ожирения; без сахарного диабета; без аденомы гипофиза; без синдрома ИценкоКушинга.

Контрольную группу составили лица мужского пола; призывного возраста без соматической патологии.

\section{Методы регистрации исходов}

Забор венозной крови для лабораторных исследований проводился в утренние часы натощак. Определение концентрации АЛТ, АСТ, глюкозы, холестерина, липидных фракций крови проводили на биохимическом анализатоpe Architect c 8000 (Abbot, США). Базальную концентрацию иммунореактивного инсулина проводили на иммунохимическом анализаторе «Architect i 2000» (Abbott, США). Определение концентрации кортизола, тестостерона, пролактина проводили на иммунохимическом анализаторе Cobas e 411 (Roche, Швейцария) с помощью набора реагентов «Алкор-Био» (СПб, Россия). Для изучения циркадной динамики кортизола его уровень определялся дважды - в 7.00 и в 21.00. Референсные значения определялись на основании данных локальной лаборатории. Для оценки функционального состояния кардиоваскулярной системы проводилось суточное мониторирование артериального давления на приборе «Кардиотехника» (ИНКАРТ, г. Санкт-Петербург) по стандартной методике [20] и эхокардиография на ультразвуковом сканере «SIEMENS G60» (Германия) по общепринятой методике [21].

Исследование функции сосудистого эндотелия проводили методом ультразвукового сканирования и с помощью контурного анализа пульсовой волны. Ультразвуковое исследование выполнено на сканере «Samsung Medison Accuvix XG» (Южная Корея) с использованием линейного мультичастотного датчика. Для оценки функционального состояния эндотелия применялась методика, предложенная D.S. Celermajer et al. (1992) [22]. Данная методика определения эндотелиальной функции основана на оценке способности эндотелия продуцировать оксид азота в условиях реактивной гиперемии и изменения кровотока. Первоначально проводили пробу с реактивной гиперемией. Для этого выполняли ультразвуковое исследование плечевой артерии и определяли ее диаметр в покое. Реактивная гиперемия модулировалась манжеточной окклюзией плечевой артерии на 4-5 мин. С помощью манжеты сфигмоманометра проводили компрессию плечевой артерии, создавая давление на 50 мм рт. ст. превышающее собственное систолическое АД пациента. Сразу же после снятия манжеты, а потом через 30 секунд и через 60 секунд вновь измеряли диаметр плечевой артерии. 
По результатам выполненных измерений рассчитывали показатель эндотелийзависимой вазодилатации (ЭЗВД, потокзависимая вазодилатация). В норме плечевая артерия расширяется на $\geq 10 \%$ на фоне реактивной гиперемии от исходного диаметра. Меньшая степень расширения или сужение сосуда считается проявлением дисфункции эндотелия. Через 10 минут отдыха пробу вновь повторяли, но с сублингвальным приемом 1 таблетки нитроглицерина. Реактивность гладкомышечного слоя сосуда оценивали по показателю эндотелийнезависимой вазодилатации (ЭНЗВД). Референсные значения для показателя ЭЗВД и ЭНЗВД составляют $\geq 10 \%$.

Контурный анализ пульсовой волны проводился на приборе «АнгиоСкан-01» (Россия). Аппарат «АнгиоСкан-01»- устройство с излучателем инфракрасных волн и датчиком, считывающим эти волны. В контексте исследования на приборе «АнгиоСкан-01» эндотелиальная дисфункция рассматривалась как нарушение нормального тока крови по сосудам вследствие снижения выработки эндотелием оксид азота. Прибор устанавливается на концевой фаланге указательного пальца верхней конечности. Инфракрасные волны проходят сквозь концевую фалангу указательного пальца верхней конечности, после чего прибор считывает их колебания и производит анализ данных в автоматическом режиме. Исследование проводилось натощак в утренние часы, перед процедурой пациенты не курили и не употребляли кофе. Анализ проводился в тихом, затемненном помещении, при температуре 20-22 ${ }^{\circ}$. При проведении исследования пациенты находились в положении сидя, кисти их рук с фотоплетизмографическими датчиками были неподвижны. С помощью прибора «АнгиоСкан» оценивались частота пульса, возраст сосудистой стенки, тип пульсовой волны, жесткость сосудистой стенки, индекс стресса, насыщение крови кислородом. Определялся показатель региональной жесткости артериального русла - индекс аугментации, нормализованный до чСС=75 уд/мин. (Alp75, augmentation index). Индекс стресса (индекс напряжения регуляторных систем, индекс Баевского) рассчитывается по показателям вариабельности ритма сердца. Данный показатель характеризует напряженность сердечно-сосудистой и дыхательной систем. Возраст сосудистой системы - показатель, характеризующий соответствие сосудистой стенки пациента, функциональному состоянию сосудистой стенки вне патологических состояний. Данный показатель определяется на основании возрастного индекса, рассчитанного с использованием математических моделей [23].

\section{Этическая экспертиза}

Протокол исследования одобрен на заседании этического комитета ФГБОУ ВО «Санкт-Петербургский государственный педиатрический медицинский университет» (протокол №01123 от 01.11.2017).

\section{Статистический анализ}

Принципы расчета размера выборки: размер выборки предварительно не рассчитывался.

Методы статистического анализа данных: Статистический анализ осуществлялся с помощью пакета программ Statistica 10.0 (StatSoftlnc, США). Нормальность распределения количественных признаков оценивалась с помощью критерия Шапиро-Уилка. При описании нормально распределенных переменных указывалось среднее и стандартное отклонение (M $\pm \sigma)$. Проверка гипотез о равенстве двух средних для параметрических данных производилась с помощью t-критерия Стьюдента. Статистически значимыми считали различия при $\mathrm{p}<0,05$. Категориальные переменные представлены в виде частоты выявления и/или в виде долей в процентах. Различия считались статистически значимыми при $p<0,05$. По коэффициенту Спирмена оценивали силу связи между переменными (r). При значениях коэффициента $r<0,3$ связь считалась слабой и незначительной; при значениях $\mathrm{r}=0,3-0,7$ - средней или умеренной; при значениях $r>0,7$ - значительной и сильной.

\section{РЕЗУЛЬТАТЫ}

\section{Объекты (участники) исследования}

Клиническая характеристика участников исследования представлена в таблице 1. Видно, что группы неоднородны по состоянию жирового и углеводного обменов, гормональному статусу. Пациенты с гипоталамическим синдромом моложе больных конституционально-экзогенным ожирением, но при этом у них наблюдаются более значимые гемодинамические, гормональные и метаболические изменения. У всех пациентов, имевших на коже розовые стрии, дебют ожирения приходился на детский возраст.

\section{Основные результаты исследования}

Результаты определения эндотелийзависимой вазодилатации (ЭЗВД) и эндотелийнезависимой вазодилатации (ЭНЗВД) у участников исследования представлены в таблице 2. Установлено, что в группе пациентов с гипоталамическим синдромом ЭЗВД ниже, чем при конституционально-экзогенном ожирении $(p<0,01)$ и в контрольной группе ( $<<0,01)$. При этом среднегрупповое значение ЭЗВД, зарегистрированное у пациентов с гипоталамическим синдромом, оказалось ниже нормы. Вместе с тем среднее значение показателя ЭНЗВД во всех группах пациентов соответствовало норме, однако в группе пациентов с гипоталамическим синдромом этот показатель оказался ниже, чем при конституционально-экзогенном ожирении $(p<0,01)$ и в контрольной группе $(p<0,01)$.

В таблице 3 представлены результаты корреляционного анализа, свидетельствующие о том, что состояние ЭЗВД может определяться множеством факторов, в числе которых возраст; повышение АД; дислипидемия; нарушение жирового и углеводного обменов; усиление продукции вазопрессорных гормонов; вялотекущее воспаление (С-реактивный белок); повышение жесткости сосудистой стенки (индекс аугментации). При этом выявлена средняя по силе корреляционная связь эндотелийзависимой вазодилатации с показателями объема талии $(r=-0,55 ; p<0,05)$ и объема бедер $(r=-0,56 ; p<0,05)$.

Анализ результатов контурного анализа пульсовой волны продемонстрировал статистически значимое увеличение индекса аугментации в группах больных с гипоталамическим синдромом и конституционально-экзогенным ожирением по сравнению с контролем (таблица 4). Среднегрупповое значение индекса аугментации у пациентов с гипоталамическим синдромом выше, чем при конституционально-экзогенном ожирении $(15,47 \pm 2,58$

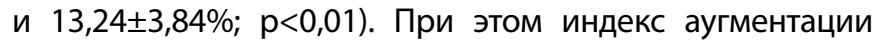


Таблица 1. Клиническая характеристика обследованных лиц.

\begin{tabular}{|c|c|c|c|c|}
\hline \multirow[b]{2}{*}{ Показатели } & \multicolumn{3}{|c|}{ Группы обследованных } & \multirow[b]{2}{*}{$\mathbf{p}$} \\
\hline & $\begin{array}{c}1 \text { грyпna } \\
(n=242)\end{array}$ & $\begin{array}{c}2 \text { группа } \\
(\mathrm{n}=98)\end{array}$ & $\begin{array}{l}\text { Контроль } \\
(n=20)\end{array}$ & \\
\hline Возраст, годы & $20,84 \pm 2,24$ & $22,18 \pm 2,48$ & $21,9 \pm 3,3$ & $\begin{array}{l}\mathrm{p}_{\mathrm{K}-1}=0,052 \\
\mathrm{p}_{\mathrm{K}-2}=0,659 \\
\mathrm{p}_{1-2}=0,001\end{array}$ \\
\hline Систолическое АД, мм рт. ст. & $150,89 \pm 18,46$ & $139,18 \pm 19,13$ & $121,76 \pm 11,43$ & $\begin{array}{l}\mathrm{p}_{\mathrm{K}-1}=0,001 \\
\mathrm{p}_{\mathrm{K}-2}=0,001 \\
\mathrm{p}_{1-2}=0,001\end{array}$ \\
\hline Диастолическое АД, мм рт. ст. & $92,16 \pm 7,68$ & $88,57 \pm 8,42$ & $81,36 \pm 9,45$ & $\begin{array}{l}p_{k-1}=0,001 \\
p_{K-2}=0,001 \\
p_{1-2}=0,001\end{array}$ \\
\hline Индекс массы тела, кг/м² & $39,79 \pm 2,68$ & $38,66 \pm 2,59$ & $23,41 \pm 3,44$ & $\begin{array}{l}\mathrm{p}_{\mathrm{K}-1}=0,001 \\
\mathrm{p}_{\mathrm{K}-2}=0,001 \\
\mathrm{p}_{1-2}=0,001\end{array}$ \\
\hline Объем талии, см & $142,17 \pm 20,08$ & $135,91 \pm 18,90$ & $62,10 \pm 3,42$ & $\begin{array}{l}p_{K-1}=0,001 \\
p_{K-2}=0,001 \\
p_{1-2}=0,010\end{array}$ \\
\hline Объем бедер, см & $116,02 \pm 15,56$ & $116,69 \pm 17,42$ & $83,80 \pm 6,05$ & $\begin{array}{l}\mathrm{p}_{\mathrm{K}-1}=0,001 \\
\mathrm{p}_{\mathrm{K}-2}=0,001 \\
\mathrm{p}_{1-2}=0,805\end{array}$ \\
\hline $\begin{array}{l}\text { Объем } \\
\text { талии/объем бедер }\end{array}$ & $1,25 \pm 0,25$ & $1,18 \pm 0,19$ & $0,74 \pm 0,02$ & $\begin{array}{l}p_{k-1}=0,001 \\
p_{K-2}=0,001 \\
p_{1-2}=0,023\end{array}$ \\
\hline Глюкоза, ммоль/л & $6,04 \pm 0,59$ & $4,98 \pm 0,93$ & $4,60 \pm 0,29$ & $\begin{array}{l}\mathrm{p}_{\mathrm{K}-1}=0,001 \\
\mathrm{p}_{\mathrm{K}-2}=0,036 \\
\mathrm{p}_{1-2}=0,001\end{array}$ \\
\hline Гликированный гемоглобин, \% & $5,65 \pm 0,72$ & $5,31 \pm 0,67$ & $4,63 \pm 0,41$ & $\begin{array}{l}p_{K-1}=0,001 \\
p_{K-2}=0,001 \\
p_{1-2}=0,001\end{array}$ \\
\hline Инсулин, мкМЕ/мл & $32,17 \pm 22,18$ & $23,81 \pm 12,91$ & $5,68 \pm 0,88$ & $\begin{array}{l}p_{K-1}=0,001 \\
p_{K-2}=0,001 \\
p_{1-2}=0,001\end{array}$ \\
\hline Индекс НОМА & $8,92 \pm 0,85$ & $5,50 \pm 0,50$ & $1,16 \pm 0,19$ & $\begin{array}{l}\mathrm{p}_{\mathrm{K}-1}=0,001 \\
\mathrm{p}_{\mathrm{K}-2}=0,001 \\
\mathrm{p}_{1-2}=0,013\end{array}$ \\
\hline Общий тестостерон, нмоль/л & $15,50 \pm 5,59$ & $16,75 \pm 6,53$ & $32,65 \pm 6,90$ & $\begin{array}{l}p_{K-1}=0,001 \\
p_{K-2}=0,001 \\
p_{1-2}=0,038\end{array}$ \\
\hline Кортизол в 7.00, нмоль/л & $361,86 \pm 49,0$ & $305,18 \pm 32,75$ & $248,80 \pm 58,37$ & $\begin{array}{l}\mathrm{p}_{\mathrm{K}-1}=0,001 \\
\mathrm{p}_{\mathrm{K}-2}=0,001 \\
\mathrm{p}_{1-2}=0,003\end{array}$ \\
\hline Кортизол в 21.00, нмоль/л & $245,59 \pm 11,44$ & $193,48 \pm 10,26$ & $106,96 \pm 10,31$ & $\begin{array}{l}\mathrm{p}_{\mathrm{K}-1}=0,001 \\
\mathrm{p}_{\mathrm{K}-2}=0,001 \\
\mathrm{p}_{1-2}=0,007\end{array}$ \\
\hline Пролактин, мМЕ/л & $356,39 \pm 29,07$ & $278,85 \pm 26,65$ & $222,30 \pm 23,75$ & $\begin{array}{l}\mathrm{p}_{\mathrm{K}-1}=0,001 \\
\mathrm{p}_{\mathrm{K}-2}=0,001 \\
\mathrm{p}_{1-2}=0,001\end{array}$ \\
\hline $\begin{array}{l}\text { Фолликулостимулирующий } \\
\text { гормон, мМЕд/мл }\end{array}$ & $4,16 \pm 2,90$ & $4,13 \pm 2,59$ & $3,25 \pm 2,05$ & $\begin{array}{l}\mathrm{p}_{\mathrm{K}-1}=0,169 \\
\mathrm{p}_{\mathrm{K}-2}=0,160 \\
\mathrm{p}_{1-2}=0,945\end{array}$ \\
\hline $\begin{array}{l}\text { Лютеинизирующий гормон, } \\
\text { мМЕд/мл }\end{array}$ & $7,43 \pm 4,40$ & $5,68 \pm 2,65$ & $1,86 \pm 0,52$ & $\begin{array}{l}p_{k-1}=0,001 \\
p_{K-2}=0,001 \\
p_{1-2}=0,001\end{array}$ \\
\hline Эстрадиол, пмоль/л & $107,69 \pm 53,21$ & $108,95 \pm 53,68$ & $60,8 \pm 23,85$ & $\begin{array}{l}\mathrm{p}_{\mathrm{K}-1}=0,001 \\
\mathrm{p}_{\mathrm{K}-2}=0,001 \\
\mathrm{p}_{1-2}=0,860\end{array}$ \\
\hline
\end{tabular}

Примечание. АД - артериальное давление.

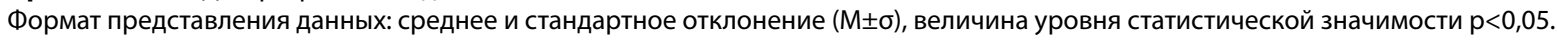


Таблица 2. Показатели эндотелийзависимой и эндотелийнезависимой вазодилатации у пациентов с гипоталамическим синдромом, конституционально-экзогенным ожирением и в контрольной группе.

\begin{tabular}{|c|c|c|c|c|c|}
\hline \multirow[b]{2}{*}{ Показатели } & \multicolumn{3}{|c|}{ Обследованные группы } & \multirow[b]{2}{*}{$\begin{array}{c}\text { Референсные } \\
\text { значения }\end{array}$} & \multirow[b]{2}{*}{$\mathbf{p}$} \\
\hline & $\begin{array}{c}1 \text { грyпna } \\
(n=242)\end{array}$ & $\begin{array}{c}2 \text { группа } \\
(n=98)\end{array}$ & $\begin{array}{c}\begin{array}{c}\text { Контроль } \\
(\mathbf{n}=20)\end{array} \\
\end{array}$ & & \\
\hline ЭЗВД, \% & $9,44 \pm 1,26$ & $10,37 \pm 1,21$ & $13,51 \pm 1,15$ & $\geq 10 \%$ & $\begin{array}{l}p_{K-1}=0,001 \\
p_{K-2}=0,001 \\
p_{1-2}=0,001\end{array}$ \\
\hline ЭНЗВД, \% & $10,29 \pm 1,28$ & $11,29 \pm 1,14$ & $14,48 \pm 1,16$ & $\geq 10 \%$ & $\begin{array}{l}p_{K-1}=0,001 \\
p_{K-2}=0,001 \\
p_{1-2}=0,001\end{array}$ \\
\hline
\end{tabular}

Примечание. ЭЗВД - эндотелийзависимая вазодилатация; ЭНЗВД - эндотелийнезависимая вазодилатация.

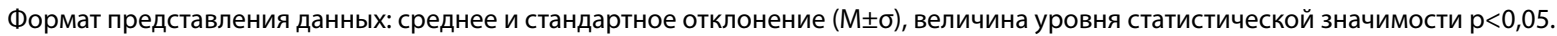

Таблица 3. Статистически значимые корреляции между эндотелийзависимой вазодилатацией и гемодинамическими, гормональными, метаболическими, воспалительными изменениями у больных ожирением

\begin{tabular}{|c|c|c|}
\hline Переменная, коррелирующая с ЭЗВД & Коэффициент корреляции Пирсона (r) & $\mathbf{p}$ \\
\hline Возраст, годы & $-0,37$ & $<0,05$ \\
\hline Систолическое АД, мм рт. ст. & $-0,41$ & $<0,05$ \\
\hline Диастолическое АД, мм рт. ст. & $-0,39$ & $<0,05$ \\
\hline Масса миокарда, г & $-0,19$ & $<0,05$ \\
\hline Индекс аугментации (Alp75), \% & $-0,42$ & $<0,05$ \\
\hline Индекс массы тела, кг/м² & $-0,61$ & $<0,05$ \\
\hline Объем талии, см & $-0,55$ & $<0,05$ \\
\hline Объем бедер, см & $-0,56$ & $<0,05$ \\
\hline Объем талии/объем бедер & $-0,35$ & $<0,05$ \\
\hline Глюкоза натощак, ммоль/л & $-0,22$ & $<0,05$ \\
\hline Гликированный гемоглобин, \% & $-0,29$ & $<0,05$ \\
\hline Инсулин, мкЕД/мЛ & $-0,37$ & $<0,05$ \\
\hline Индекс НОМА & $-0,37$ & $<0,05$ \\
\hline Общий холестерин, ммоль/л & $-0,37$ & $<0,05$ \\
\hline ЛПНП, ммоль/л & $-0,29$ & $<0,05$ \\
\hline ЛПОНП, ммоль/л & $-0,53$ & $<0,05$ \\
\hline Триглицериды, ммоль/л & $-0,23$ & $<0,05$ \\
\hline Коэффициент атерогенности & $-0,24$ & $<0,05$ \\
\hline Кортизол в 21.00, нмоль/л & $-0,23$ & $<0,05$ \\
\hline Пролактин, мМЕ/л & $-0,23$ & $<0,05$ \\
\hline Лютеинизирующий гормон, МЕ/мл & $-0,37$ & $<0,05$ \\
\hline Тестостерон, нмоль/л & 0,46 & $<0,05$ \\
\hline С-реактивный белок, г/л & $-0,19$ & $<0,05$ \\
\hline
\end{tabular}

Примечание. АД - артериальное давление; ЛПОНП - липопротеиды очень низкой плотности; ЛПНП - липопротеиды низкой плотности.

Таблица 4. Результаты контурного анализа пульсовой волны у пациентов с гипоталамическим синдромом, конституциональноэкзогенным ожирением и в контрольной группе.

\begin{tabular}{|c|c|c|c|c|c|}
\hline \multirow[b]{2}{*}{ Показатели } & \multicolumn{3}{|c|}{ Обследованные группы } & \multirow[b]{2}{*}{$\begin{array}{c}\text { Референсные } \\
\text { значения }\end{array}$} & \multirow[b]{2}{*}{$\mathbf{p}$} \\
\hline & $\begin{array}{l}1 \text { групnа } \\
(n=242)\end{array}$ & $\begin{array}{c}2 \text { группа } \\
(n=98)\end{array}$ & $\begin{array}{c}\text { Контроль } \\
(n=20)\end{array}$ & & \\
\hline $\begin{array}{l}\text { Индекс аугментации (Alp75), } \\
\%\end{array}$ & $15,47 \pm 2,58$ & $13,24 \pm 3,84$ & $7,26 \pm 2,39$ & $\begin{array}{l}\text { от }-12,0 \\
\text { до } 8,0\end{array}$ & $\begin{array}{l}p_{K-1}=0,001 \\
p_{K-2}=0,001 \\
p_{1-2}=0,001\end{array}$ \\
\hline $\begin{array}{l}\text { Биологический возраст } \\
\text { сосудистой стенки, годы }\end{array}$ & $43,57 \pm 6,26$ & $43,12 \pm 6,91$ & $21,91 \pm 3,42$ & $\begin{array}{l}\text { Фактический } \\
\text { возраст }\end{array}$ & $\begin{array}{l}p_{K-1}=0,001 \\
p_{K-2}=0,001 \\
p_{1-2}=0,812\end{array}$ \\
\hline $\begin{array}{l}\text { Индекс стресса } \\
\text { (индекс Баевского) }\end{array}$ & $837,15 \pm 149,54$ & $874,13 \pm 188,04$ & $75,69 \pm 12,45$ & $50-150$ & $\begin{array}{l}p_{K-1}=0,001 \\
p_{K-2}=0,001 \\
p_{1-2}=0,531\end{array}$ \\
\hline
\end{tabular}

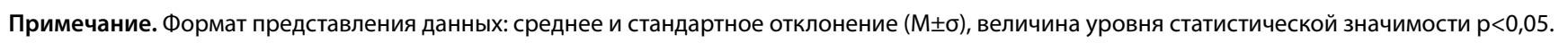


в группах пациентов с ожирением в 1,8 раза выше, чем в контрольной группе. Средний возраст сосудистой стенки, рассчитанный в группе пациентов с гипоталамическим синдромом и конституционально-экзогенным ожире-

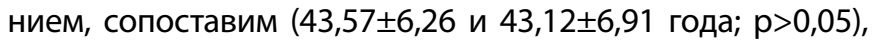
но в 2 раза выше, чем в контрольной группе $(43,57 \pm 6,26$

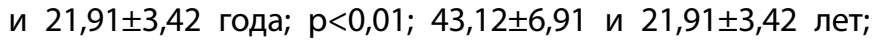
$\mathrm{p}<0,01)$. Группы пациентов с гипоталамическим синдромом и конституционально-экзогенным ожирением оказались сопоставимы по индексу стресса, отражающему активность симпатического отдела вегетативной нервной системы $(837,15 \pm 149,54$ и 874,13 $\pm 188,04 ;$ р>0,05). Среднее значение индекса стресса в 1 и 2 группах в 10 раз выше, чем в контрольной группе ( $p<0,01)$.

В таблице 5 представлены статистически значимые корреляции между возрастом сосудистой стенки и гемодинамическими, гормональными, метаболическими изменениями у больных ожирением. Установлено, что интенсивность ангиологического старения определяется такими гемодинамическими факторами, как увеличение чСС $(r=0,32 ; p<0,05)$; повышение уровня среднесуточного систолического АД ( $r=0,27 ; p<0,05)$; повышение уровня среднесуточного диастолического АД $(r=0,22 ; p<0,05)$; увеличение вариабельности систолического АД в дневное время $(r=0,25 ; p<0,001)$; увеличение вариабельности диастолического АД в дневное время $(r=0,17 ; \mathrm{p}<0,05)$. Среди метаболических факторов, определяющих интенсивность ангиологического старения периферических артерий, следует отметить повышение ИМТ
( $\mathrm{r}=0,23 ; \mathrm{p}<0,05) ;$ увеличение инсулинорезистентности $(r=0,17 ; p<0,05)$; атерогенность липидного спектра с повышением концентрации липопротеидов очень низкой плотности ( $r=0,39 ; p<0,05)$. Структурно-функциональное состояние сосудистой стенки также связано с повышением C-реактивного белка ( $r=0,27 ; p<0,05)$, что отражает участие воспалительных механизмов в ухудшении функции эндотелия и прогрессировании процессов ангиологического старения. Выявлена слабая по силе корреляционная связь между интенсивностью процессов ангиологического старения и снижением ЭЗВД ( $r=-0,31$; $\mathrm{p}<0,05)$, а также снижением ЭНЗВД $(r=-0,33 ; \mathrm{p}<0,05)$. Доказана высокая значимость активации симпатического отдела вегетативной нервной системы в интенсивности процессов ангиологического старения (индекс стресса $r=0,51 ; p<0,05)$. Получены данные о существовании прямой корреляционной связи между уровнем трансаминаз (АЛТ, АСТ) и сосудистым возрастом. Данный факт можно объяснить лишь тем, что повышение АЛТ и АСТ обусловлено жировым гепатозом, поскольку данная патология наблюдалась у большинства больных ожирением независимо от его этиологии $(91,7 \%)$, а уровень трансаминаз был достоверно выше в группе с гипотала-

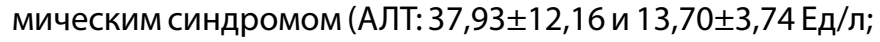
$\mathrm{p}=0,001 ;$ АСТ: 29,65 $\pm 13,08$ и 16,30 $\pm 2,54$ Ед/л; $p=0,001)$ и с конституционально-экзогенным ожирением (АЛТ:

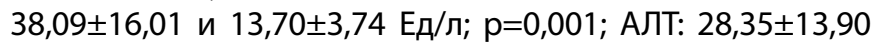

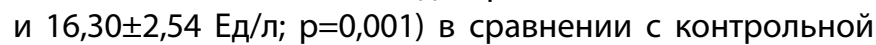
группой. Таким образом, выполненный корреляционный

Таблица 5. Статистически значимые корреляции между биологическим возрастом сосудистой стенки и гемодинамическими, гормональными, метаболическими, воспалительными изменениями у больных ожирением

\begin{tabular}{lcc}
\hline \multicolumn{1}{c}{$\begin{array}{c}\text { Переменная, коррелирующая с биологическим возрастом } \\
\text { сосудистой стенки }\end{array}$} & $\begin{array}{c}\text { Коэффициент корреляции } \\
\text { Пирсона (r) }\end{array}$ & p \\
\hline ЧСС, уд/мин & 0,32 & $<0,05$ \\
Среднесуточное систолическое АД, мм рт. ст. & 0,27 & $<0,05$ \\
Среднесуточное диастолическое АД, мм рт. ст. & 0,22 & $<0,05$ \\
Вариабельность систолического АД в дневное время, мм рт. ст. & 0,25 & $<0,05$ \\
Вариабельность диастолического АД в дневное время, мм рт. ст. & 0,17 & $<0,05$ \\
Относительная толщина стенки левого желудочка & 0,23 & $<0,05$ \\
Индекс массы тела, кг/м & 0,23 & $<0,05$ \\
Индекс НОМА & 0,17 & $<0,05$ \\
ЛПОНП, ммоль/л & 0,36 & $<0,05$ \\
ЛПНП, ммоль/л & 0,39 & $<0,05$ \\
Коэффициент атерогенности & 0,26 & $<0,05$ \\
С-реактивный белок, г/л & 0,27 & $<0,05$ \\
ЭЗВД, \% & $-0,31$ & $<0,05$ \\
ЭНЗВД, \% & $-0,33$ \\
Индекс стресса (индекс Баевского) & $<0,05$ \\
Индекс аугментации (АІр75), \% & 0,51 & $<0,05$ \\
АЛТ, Ед & 0,45 & $<0,05$ \\
АСТ, Ед & 0,05 \\
\hline
\end{tabular}

Примечание. чСС - частота сердечных сокращений; АД - артериальное давление; лпОНП - липопротеиды очень низкой плотности; лПнП липопротеиды низкой плотности; ЭЗВД - эндотелийзависимая вазодилатация; ЭНЗВД - эндотелийнезависимая вазодилатация; АСТ - аланинаминотрансфераза; АЛТ - аспартатаминотрансфераза. 
Таблица 6. Статистически значимые корреляции между жесткостью сосудистой стенки и гемодинамическими, гормональными, метаболическими, воспалительными изменениями у больных ожирением.

\begin{tabular}{|c|c|c|}
\hline Переменная, коррелирующая с индексом аугментации & $\begin{array}{c}\text { Коэффициент корреляции } \\
\text { Пирсона (r) }\end{array}$ & $\mathbf{p}$ \\
\hline Среднесуточное систолическое АД, мм рт. ст. & 0,21 & $<0,05$ \\
\hline Среднесуточное диастолическое АД, мм рт. ст. & 0,19 & $<0,05$ \\
\hline Вариабельность систолического АД в дневное время, мм рт. ст. & 0,24 & $<0,05$ \\
\hline Вариабельность диастолического АД в дневное время, мм рт. ст. & 0,18 & $<0,05$ \\
\hline 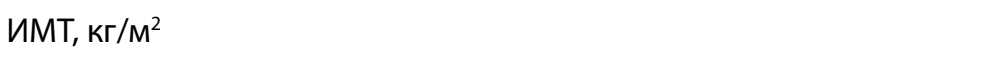 & 0,18 & $<0,05$ \\
\hline Гликированный гемоглобин, \% & 0,29 & $<0,05$ \\
\hline Индекс НОМА & 0,17 & $<0,05$ \\
\hline Коэффициент атерогенности & 0,26 & $<0,05$ \\
\hline лПОНП, ммоль/л & 0,36 & $<0,05$ \\
\hline лПНП, ммоль/л & 0,39 & $<0,05$ \\
\hline С-реактивный белок, г/л & 0,36 & $<0,05$ \\
\hline ЭЗВД, \% & $-0,17$ & $<0,05$ \\
\hline ЭНЗВД, \% & $-0,18$ & $<0,05$ \\
\hline
\end{tabular}

Примечание. АД - артериальное давление; ИМТ - индекс массы тела; ЛПОНП - липопротеиды очень низкой плотности; лПнП - липопротеиды низкой плотности; ЭЗВД - эндотелийзависимая вазодилатация; ЭНЗВД - эндотелийнезависимая вазодилатация.

анализ свидетельствует о существенном вкладе гормональных, метаболических и гемодинамических нарушений в прогрессирование структурно-функциональных изменений сосудистой стенки у больных ожирением независимо от его этиологии.

В таблице 6 представлены результаты корреляционного анализа, отражающие связь между сосудистой жесткостью и гемодинамическими, гормональными, метаболическими изменениями у больных ожирением. Установлено, что жесткость сосудистой стенки связана с повышением уровня среднесуточного систолического АД $(r=0,21 ; p<0,05)$; среднесуточного диастолического АД $(r=0,19 ; p<0,05)$; вариабельности систолического АД в дневное время $(r=0,24 ; p<0,001)$; вариабельности диастолического АД в дневное время $(r=0,18 ; p<0,05)$, что весьма закономерно. Также получены данные о том, что жесткость сосудистой стенки связана с нарушением углеводного обмена, дислипидемией, повышением ИТМ, эндотелиальной дисфункцией, вялотекущим воспалением (С-реактивный белок).

\section{Дополнительные результаты исследования}

У пациентов с гипоталамическим синдромом зафиксирован более высокий уровень общего холестерина, чем при конституционально-экзогенном ожирении $(5,01 \pm 1,06$ и 4,69 $\pm 0,86$ ммоль/л; $p=0,008)$ и в контрольной группе $(5,01 \pm 1,06$ и 3,59 $\pm 0,57 ; p=0,001)$. Помимо этого у пациентов с гипоталамическим синдромом отмечался более высокий коэффициент атерогенности в сравнении с конституционально-экзогенным ожирением $(3,05 \pm 0,71$

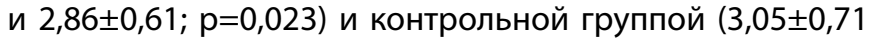
и 2,27 $\pm 0,18 ; p=0,001)$. Другие показатели липидного спектра были сопоставимы между пациентами с гипоталамическим синдромом и конституционально-экзогенным ожирением. Это касалось уровня липопротеидов низкой плотности $(3,26 \pm 0,67$ и 3,41 00,77 ммоль/л; $p=0,071)$, липопротеидов очень низкой плотности $(0,62 \pm 0,27$

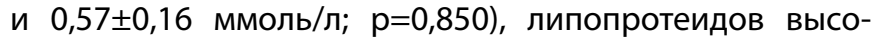
кой плотности $(1,24 \pm 0,39$ и 1,29 $\pm 0,32$ ммоль/л; $\mathrm{p}=0,380)$ и триглицеридов $(1,53 \pm 0,56$ и 1,53 $\pm 0,65$ ммоль/л; $\mathrm{p}=0,985)$. Таким образом, у пациентов с гипоталамическим синдромом профиль липидов крови оказался более атерогенным, чем у больных конституционально-экзогенным ожирением. Корреляционный анализ показал, что повышение атерогенности липидного спектра крови может быть связано с нарушением углеводного, жирового и гормонального обменов. Так значение коэффициента атерогенности находится в прямой корреляционной связи с концентрацией гликированного гемоглобина $(r=0,18 ; p<0,05)$; индексом НОМА $(r=0,12 ; p<0,05)$; инсулином $(r=0,26 ; p<0,05)$; соотношением объем талии/объем бедер ( $r=0,52 ; \mathrm{p}<0,05) ;$ пролактином $(r=0,17 ; \mathrm{p}<0,05)$; лютеинизирующим гормоном $(r=0,24 ; p<0,05)$.

Особенностью пациентов с гипоталамическим синдром являлся более высокий уровень С-реактивного белка, чем при конституционально-экзогенном ожирении $(6,50 \pm 0,24$ и 3,91 $\pm 0,36$ г/л; $p=0,001)$ и в контрольной группе $(6,50 \pm 0,24$ и 3,21 $\pm 0,76$ г/л; $p=0,001)$. Установлены статистически значимые корреляции между уровнем С-реактивного белка и ИМТ $(r=0,21 ; \mathrm{p}<0,05)$; объемом талии ( $r=0,27 ; p<0,05)$; объемом бедер $(r=0,18 ; p<0,05)$; глюкозой $(r=0,26 ; p<0,05) ;$ гликированным гемоглобином $(r=0,21 ; p<0,05)$; инсулином $(r=0,18 ; p<0,05)$; липопротеидами высокой плотности $(r=-0,26 ; p<0,05)$; липопротеидами очень низкой плотности $(r=0,29 ; p<0,05)$; триглицеридами $(r=0,21 ; p<0,05) ;$ индексом аугментации $(r=0,36$; $\mathrm{p}<0,05)$; ЭЗВД $(r=-0,19 ; \mathrm{p}<0,05) ;$ ЭНЗВД $(r=-0,21 ; p<0,05)$; возрастом сосудистой стенки $(r=0,27 ; p<0,05)$. Помимо этого повышение С-реактивного белка может быть связано и изменением функциональной активности системы гипоталамус-гипофиз-надпочечники. Доказательством этому являются установленные корреляции между повышением C-реактивного белка и усилением продукции кортизола в $7.00(r=0,19 ; p<0,05) ;$ кортизола в 21.00 
$(r=0,28 ; p<0,05) ;$ пролактина $(r=0,17 ; p<0,05)$. Подобные гормональные изменения свойственны пациентам с гипоталамическим синдромом.

Нежелательные явления

В ходе проведения исследования нежелательных явлений не зарегистрировано.

\section{ОБСУЖДЕНИЕ}

\section{Резюме основного результата исследования}

У пациентов с гипоталамическим синдромом и конституционально-экзогенным ожирением показатели жесткости сосудистой стенки, индекса стресса, ангиологического возраста достоверно выше, а показатели ЭЗВД и ЭНЗВД достоверно ниже, чем в контрольной группе. Более выраженные структурно-функциональные изменения сосудистой стенки отмечались у пациентов с гипоталамическим синдромом. У больных ожирением нарушение архитектоники сосудистой стенки, прогрессирование артериальной ригидности и интенсивность ангиологического старения обусловлены выраженностью гормональных, метаболических и гемодинамических нарушений, а также активностью вялотекущих воспалительных процессов.

\section{Обсуждение основного результата исследования}

В России на протяжении многих лет термин «гипоталамический синдром пубертатного периода» являлся часто используемым и наиболее оправданным, так как именно он в полной мере отражает топику поражения при данной патологии [24]. Однако в последние годы нейроэндокриннообменный синдром все чаще обозначают как «метаболический синдром», не учитывая при этом нарушений в репродуктивной системе, возникающих при гипоталамическом синдроме пубертатного периода [25]. За рубежом гипоталамический синдром пубертатного периода не принято выделять в отдельную нозологическую форму. При этом розовые стрии, являющиеся патогномоничным признаком гипоталамического синдрома пубертатного периода, принимаются за симптом дерматологического заболевания. Вместе с тем российские ученые полагают, что появление розовых стрий, прежде всего, обусловлено дисфункцией гипоталамуса и усилением продукции кортизола [26]. В более ранних исследованиях нами были продемонстрированы более выраженные гормональные, метаболические и гемодинамические нарушения у пациентов с гипоталамическим синдромом (ожирение с розовыми стриями), чем при конституционально-экзогенном ожирении [27, 28], что доказывает целесообразность рассмотрения гипоталамического синдрома с позиции отдельно выделенной нейроэндокриннообменной патологии.

До сих пор не выработано единого мнения о том, какой подход к оценке эндотелиальной функции можно считать наилучшим, а референсные значения каждого метода являются произвольными и несопоставимыми. В своей работе мы использовали неинвазивные, недорогостоящие и простые в применении методы оценки эндотелиальной функции.

В настоящем исследовании установлена связь гемодинамических, гормональных, метаболических, воспалительных изменений, возникающих у юношей с гипо- таламическим синдромом, с развитием эндотелиальной дисфункции и преждевременным ангиологическим старением. Результаты нашего исследования согласуются с данными, полученными зарубежными коллегами. В частности при изучении связи между подростковым ожирением и эндотелиальной дисфункцией установлены корреляции между соотношением окружность талии/ рост и толщиной комплекса интима-медиа сонной артерии $(r=0,23)$, а также между содержанием в сыворотке молекул межклеточной адгезии-1 и инсулинорезистентностью $(r=0,32)$ [29]. Однако в другом подобном исследовании, не выявлено различий в состоянии сосудистой стенки между подростками с ожирением и нормальным весом [30]. Возможно, это связано с тем, что в данной работе в качестве маркера эндотелиальной дисфункции оценивалась лишь толщина комплекса интима-медиа, тогда как у лиц молодого возраста показатели жесткости сосудистой стенки более информативны для оценки структурно-функционального состояния сосудов [31].

В настоящее время понятие «сердечно-сосудистого возраста» рекомендуется широко использовать при проведении профилактического консультирования пациентов, в особенности молодого возраста с низким абсолютным, но высоким относительным риском. В данном исследовании маркером интенсивности сердечно-сосудистого старения являлся показатель возраста сосудистой стенки, определяемый при контурном анализе пульсовой волны. Исследование показало, что структурно-функциональные изменения сосудистой стенки могут являться следствием влияния возраста; повышения уровня АД; жесткости сосудистой стенки; гиперсимпатикотонии; нарушений жирового и углеводного обменов; дислипидемии; усиления продукции вазопрессорных гормонов; вялотекущих воспалительных процессов. Получены доказательства существенного вклада гормональных, метаболических, гемодинамических, воспалительных изменений в нарушение архитектоники сосудистой стенки, прогрессирование артериальной ригидности и интенсивность ангиологического старения у больных ожирением независимо от его этиологии. Сдвиги гормонально-метаболического статуса, развивающиеся у пациентов юношеского и молодого возраста с гипоталамическим синдромом, обусловливают раннее развитие эндотелиальной дисфункции, и это создает предпосылки для ускоренного возникновения кардиоваскулярной патологии у этой категории больных.

\section{Резюме основного результата исследования}

Для пациентов с гипоталамическим синдромом свойственно более выраженное изменение липидного спектра крови и прогрессирующее хроническое воспаления, чем при конституционально-экзогенном ожирении. Прогрессирование вялотекущего воспаления при гипоталамическом синдроме обусловлено нарушениями углеводного и жирового обменов, а также нейрогормональными сдвигами на фоне изменения функциональной активности системы гипоталамус-гипофиз-надпочечники.

Обсуждение дополнительного результата

исследования

Результаты исследований показывают, что у больных ожирением отмечается достоверное снижение 
эндотелийзависимой вазодилатации по мере нарастания степени ожирения [32]. У пациентов с ожирением развитию эндотелиальной дисфункции способствуют множественные механизмы, в том числе дислипидемия и прогрессирующее хроническое воспаление [9, 10]. Настоящее исследование показало, что у пациентов с гипоталамическим синдромом профиль липидов обладает более атерогенными свойствами, чем при конституционально-экзогенном ожирении. В других исследованиях развитие дислипидемии при ожирении связывали столько с нарушением углеводного и жирового обменов [8], при этом последствия нейрогормональных нарушений центрального генеза в литературе не описывались. Оказалось, что повышение атерогенности липидного спектра крови может быть связано не только с нарушением углеводного и жирового обменов, но и с повышением уровня пролактина $(r=0,17 ; p<0,05)$ и лютеинизирующего гормона $(r=0,24 ; p<0,05)$, как следствие изменения функциональной активности гипоталамо-гипофизарных структур. Другим не менее значимым индуктором развития эндотелиальной дисфункции является хроническое медленно прогрессирующее воспаление [9]. При гипоталамическом синдроме уровень С-реактивного белка оказался в 2 раза выше, чем при конституционально-эк-

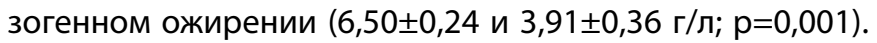
Этот факт указывает на более прогрессивный характер вяло текущего воспаления при данной нейроэндокринной патологии по сравнению с обычным конституционально-экзогенным ожирением. Прогрессирование вялотекущего воспаления при гипоталамическом синдроме может быть связано с дислипидемией, изменением структурно-функциональной структуры сосудистой стенки, нарушениями жирового и углеводного обменов, нейрогормональными сдвигами в виде усиления продукции кортизола и пролактина.

\section{Ограничения исследования}

В нашем исследовании не проводился формальный расчет объема выборки, что допустимо в рамках неинтервенционного исследования. Набор пациентов в исследование осуществлялся среди лиц мужского пола призывного возраста 18-27 лет, направленных райвоенкоматами для обследования в эндокринологическое отделение, т е. выборочным методом в соответствии с критериями включения в исследование. Жесткие критерии отбора участников исследования уменьшают вероятность влияния на результат дополнительных вмешивающихся факторов. Однако в реальной клинической практике на клиническое течение гипоталамического синдрома могут влиять факторы образа жизни, пищевое поведение, сопутствующие заболевания. Таким образом, экстраполяция результатов на общую популяцию пациентов с гипоталамическим синдромом некорректна

\section{ЗАКЛЮЧЕНИЕ}

У пациентов с гипоталамическим синдромом и конституционально-экзогенным ожирением показатели жесткости сосудистой стенки, индекса стресса, ангиологического возраста достоверно выше, а показатели ЭЗВД и ЭНЗВД достоверно ниже, чем в контрольной группе. Полученные данные свидетельствуют об ухудшении функции эндотелия и повышении жесткости сосудистой стенки у больных, страдающих ожирением, независимо от его этиологии. Однако у пациентов с гипоталамическим синдромом отмечаются более выраженные структурно-функциональные изменения сосудистой стенки, чем при конституционально-экзогенном ожирении. Получены доказательства существенного вклада гормональных, метаболических, гемодинамических, воспалительных изменений в нарушение архитектоники сосудистой стенки, прогрессирование артериальной ригидности и интенсивность ангиологического старения у больных ожирением независимо от его этиологии.

\section{ДОПОЛНИТЕЛЬНАЯ ИНФОРМАЦИЯ}

Источники финансирования. Исследование выполнено при лабораторном и инструментальном обеспечении плановой НИР с использованием ресурсов базового медицинского учреждения.

Конфликт интересов. Авторы декларируют отсутствие явных и потенциальных конфликтов интересов, связанных с содержанием настоящей статьи.

Участие авторов. Церцвадзе Лана Кахаберовна - существенный вклад в получение, анализ данных и интерпретацию результатов, написание статьи; Авдеева Марина Владимировна - существенный вклад в концепцию и дизайн исследования, внесение в рукопись существенной правки с целью повышения научной ценности статьи; Щеглова Лариса Васильевна - существенный вклад в концепцию исследования, внесение в рукопись существенной правки с целью повышения научной ценности статьи; Василенко Владимир Станиславович существенный вклад в концепцию исследования, внесение в рукопись существенной правки с целью повышения научной ценности статьи Все авторы одобрили финальную версию статьи перед публикацией, выразили согласие нести ответственность за все аспекты работы, подразумевающую надлежащее изучение и решение вопросов, связанных с точностью или добросовестностью любой части работы.

\section{СПИСОК ЛИТЕРАТУРЫ | REFERENCES}

1. GBD 2015 Obesity Collaborators. Health Effects of Overweight and Obesity in 195 Countries over 25 Years. N Engl J Med. 2017;377(1):13-27. doi: https://doi.org/10.1056/NEJMoa1614362

2. Mattsson M, Maher GM, Boland F, et al. Group-based trajectory modelling for BMI trajectories in childhood: A systematic review. Bierhaus A, ed. Obes Rev. 2019;20(7):998-1015. doi: https://doi.org/10.1111/obr.12842

3. Крук Л.П., Строев Ю.И., Чурилов Л.П. Функциональное состояние щитовидной железы при гипоталамическом синдроме пубертатного периода // Сб. «Здоровье - основа человеческого потенциала: проблемы и пути их решения». - 2018. - Т. 13. - №1.
C. 297-304. [Kruk LP, Stroev Yul, Churilov LP. Funkcional'noe sostoyanie shchitovidnoj zhelezy pri gipotalamicheskom sindrome pubertatnogo perioda [abstract]. St. Petersburg. 2018; 13 Suppl 1: 297-304. (In Russ.)]

4. Габбасова Н.В., Дзень Н.В., Хаперскова М.А. Анализ заболеваемости ожирением в Воронежской области за период 2011-2016 годы // Научно-медицинский вестник Центрального Черноземья. - 2017. - No70. - C. 11-15. [Gabbasova NV, Dzen NV, Khaperskova MA. Analysis of the incidence of obesity in the Voronezh region for the period 2011-2016. Scientific and Medical Bulletin of the Central Chernozem Region. 2017; (70): 11-15. (In Russ.)] 
5. Genovesi S, Antolini L, Giussani M, et al. Hypertension, Prehypertension, and Transient Elevated Blood Pressure in Children: Association With Weight Excess and Waist Circumference. Am J Hypertens. 2010;23(7):756-761. doi: https://doi.org/10.1038/ajh.2010.50

6. Friedemann C, Heneghan C, Mahtani $\mathrm{K}$, et al. Cardiovascular disease risk in healthy children and its association with body mass index: systematic review and meta-analysis. BMJ. 2012;345(sep25 2):e4759-e4759. doi: https://doi.org/10.1136/bmj.e4759

7. Valerio G, Maffeis C, Saggese G, et al. Diagnosis, treatment and prevention of pediatric obesity: consensus position statement of the Italian Society for Pediatric Endocrinology and Diabetology and the Italian Society of Pediatrics. Ital J Pediatr. 2018;44(1):88. doi: https://doi.org/10.1186/s13052-018-0525-6

8. Лавренкова Е.А., Драпкина О.М. Инсулинорезистентность при ожирении: причины и последствия // Ожирение и метаболизм. 2020. - T. 17. - No1. - C. 48-55. [Lavrenova EA, Drapkina OM. Insulin resistance in obesity: pathogenesis and effects. Obesity and metabolism. 2020;17(1):48-55. (In Russ.)] doi: https://doi.org/10.14341/omet9759

9. Смирнова Н.Н., Куприенко Н.Б., Жестянникова Е.И. Эндотелиальная дисфункция при ожирении у детей. - 2019. - Т. 4. - №2. - С. 35-41 // Медицина: теория и практика [Smirnova NN, Kuprienko NB, Gestjannikova El. Endothelial dysfunction in obese children. Medicine: theory and practice. 2019:4(2):35-41. (In Russ.)]

10. Чусова Н.А. Роль эндотелиальной дисфункции при ожирении // Международный студенческий научный вестник. - 2019. - №5-2. C. 67 [Chusova NA. Role of endothelial dysfunction in obesity. International student scientific bulletin. 2019;(5-2):67. (In Russ.)]

11. Nikonova L V., Tishkovskiy S V., Butrim OS, Davydchyk EV. Hypothalamic syndrome. The role of the hypothalamus in the formation of eating behavior and obesity. $J$ Grodno State Med Univ. 2019;17(4):355-360. doi: https://doi.org/10.25298/2221-8785-2019-17-4-355-360

12. Строев Ю.И., Чурилов Л.П., Али Н., и др. Ожирение с розовыми стриями - проблемная патология детей и подростков // Сб. материалов IX российского форума «Педиатрия СанктПетербурга: опыт, инновации, достижения». - 2019. - С. 166-173. [Stroev Yul, Churilov LP, Ali N, et al. Ozhirenie s rozovymi striyami problemnaya patologiya detej i podrostkov [abstract]. St. Petersburg. 2019; S.166-173. (In Russ.)]

13. Borowska-Striuk T. Therapeutic tactics in the hypothalamic syndrome of puberty in girls. Medicni perspektivi (Medical perspectives). 2018;23(3):92-97. doi: https://doi.org/10.26641/2307-0404.2018.3.147963

14. Bereket A, Kiess W, Lustig RH, et al. Hypothalamic Obesity in Children. In: Kiess W, Wabitsch M, Maffeis C, Sharma AM (eds): Metabolic Syndrome and Obesity in Childhood and Adolescence. Pediatr Adolesc Med. Basel, Karger, 2015;19:13-30. doi: https://doi.org/10.1159/000368102

15. Bereket A, Kiess W, Lustig RH, et al. Hypothalamic obesity in children. Obes Rev. 2012;13(9):780-798. doi: https://doi.org/10.1111/j.1467-789X.2012.01004.x

16. Ершевская А.Б., Новикова А.П., Лесик И.П. Патогенетические механизмы ожирения у детей // Вестник НовГУ. - 2018. - №5. C. 35-37. [Ershevskaya AB, Novikova AP, Lesik IP. Pathogenetic mechanisms of obesity in children // Bulletin of Novgorod State University. 2018;(5):35-37. (In Russ.)]

17. Отто Н.Ю., Сагитова Г.Р., Никулина Н.Ю., Ледяев М.Я. Частота метаболического синдрома и других осложнений ожирения в практике детского эндокринолога // Вестник Волгоградского государственного медицинского университета. - 2018. - Т. 67. №3. - C. 93-98. [Otto NYu, Sagitova GR, Nikulina NYu, Ledyaev MYa. The frequency of the metabolic syndrome and other complications of obesity in the practice of a pediatric endocrinologist. Bulletin of Novgorod State University. 2018;67(3):93-98. (In Russ.)]

18. Точилина О.В., Андреева И.Н. Физические факторы в комплексном лечении дисфункции гипоталамуса в подростковом возрасте // Вестник физиотерапии и курортологии. - 2017. - Т. 23. - №4. C. 181. [Tochilina OV, Andreeva IN. Physical factors in the complex treatment of hypothalamic dysfunction in adolescence. Bulletin of physiotherapy and balneology. 2017;23(4):181. (In Russ.)]

19. Oluwagbemigun K, Buyken AE, Alexy U, Schmid M, Herder C, Nöthlings U. Developmental trajectories of body mass index from childhood into late adolescence and subsequent late adolescence-young adulthood cardiometabolic risk markers. Cardiovasc Diabetol. 2019;18(1):9. doi: https://doi.org/10.1186/s12933-019-0813-5
20. Рогоза А.Н., Никольский В.П., Ощепкова Е.В. Суточное мониторирование артериального давления при гипертонии. Методические вопросы. - М., 2005. - 36 c. [Rogoza A.N., Nikol'skij V.P., Oshchepkova E.V. Sutochnoe monitorirovanie arterial'nogo davleniya pri gipertonii. Metodicheskie voprosy. - M., 2005. - 36 s. (In Russ.)]

21. Рекомендации по количественной оценке структуры и функции камер сердца // Российский кардиологический журнал. - 2012. T.95. - №3. - C. 1-28. [Recommendations for the quantitative assessment of the structure and function of the heart chambers. Russian Journal of Cardiology. 2012; 95(3): 1-28. (In Russ).]

22. Celermajer DS, Sorensen KE, Spiegelhalter DJ, et al. Aging is associated with endothelial dysfunction in healthy men years before the agerelated decline in women. J Am Coll Cardiol. 1994;24(2):471-476. doi: https://doi.org/10.1016/0735-1097(94)90305-0

23. Метод Парфенова - о необходимости оценки артериальной функции [Early diagnosis of cardiovascular disease (CVD) is the main area of our research and development In Russ.)] Доступно по: https://angioscan.ru/ru/parfenov-method/af-assessment. Ссылка активна на 17.02.2020

24. Ж Жуковец И.В., Быстрицкая Т.С., Конеева Е.Я., Путинцева О.Г. Этиология и патогенез дисфункции гипоталамуса: современный взгляд на проблему (Обзор литературы) // Бюллетень физиологии и патологии дыхания. - 2011. - №42. - С. 96-99. [Zhukovets IV, Bystritskaya TS, Koneeva EYa, Putintseva OG. Etiology and pathogenesis of hypothalamic dysfunction: a modern view of the problem (Literature review). Bulletin of physiology and respiratory pathology. 2011; (42): 96-99. (In Russ.)]

25. Жуковец И.В. Спорные вопросы нозоологии: метаболический синдром или дисфункция гипоталамуса? // Амурский медицинский журнал. - 2014. - T.5. - No1. - C. 105-108. [Zhukovets IV. Controversial issues of nosoology: metabolic syndrome or dysfunction of the hypothalamus? Amur Medical Journal. 2014;5(1):105-108. (In Russ.)]

26. Строев Ю.И., Чурилов Л.П. Кожные стрии: новые представления о патогенезе и диагностической значимости старого симптома // Медицина. XXI век. - 2008. - №11. - C. 50-55. [Stroyev Yul, Churilov LP. Skin striae: new ideas about the pathogenesis and diagnostic significance of the old symptom. Medicine. XXI Century. 2008;(11):50-55. (In Russ.)]

27. Церцвадзе Л.К., Авдеева М.В., Щеглова Л.В., Василенко В.С. Особенности гормонального и кардиометаболического профиля у юношей с гипоталамическим синдромом позднего пубертатного периода // Современные проблемы науки и образования. - 2019. - №4. [Tsertsvadze LK, Avdeeva MV, Scheglova LV, Vasilenko VS. Features of the hormonal and cardiometabolic profile in young men with the hypotalamic syndrome of the late pubertary period. Modern problems of science and education. 2019;(4):36. (In Russ.)] doi: https://doi.org/10.17513/spno.29064

28. Церцвадзе Л.К., Авдеева М.В., Щеглова Л.В., Ерина М.Ю. Предикторы метаболического синдрома у юношей и лиц молодого возраста в зависимости от степени их половозрелости и причины ожирения // Современные проблемы науки и образования. - 2019. [Tsertsvadze LK, Avdeeva MV, Scheglova LV, Erina MYu. Predictors of metabolic syndrome in youths and young men depending on the degree of purification and reason of obesity. Modern problems of science and education. 2019;(5). (In Russ.)] doi: https://doi.org/10.17513/spno.29094

29. Ribeiro ACR, Lyra A, Bonfitto AJ, et al. Assessment of intimamedia thickness of the carotid artery and intraluminal diameter of the brachial artery as cardiovascular risk markers in Brazilian adolescents with overweight or obesity. J Pediatr Endocrinol Metab 2020;33(3):339-345. doi: https://doi.org/10.1515/jpem-2019-0254

30. Murni IK, Sulistyoningrum DC, Susilowati R, Julia M. Risk of metabolic syndrome and early vascular markers for atherosclerosis in obese Indonesian adolescents. Paediatr Int Child Health. 2020;40(2):117-123. doi: https://doi.org/10.1080/20469047.2019.1697568

31. Bruyndonckx L, Hoymans VY, Van Craenenbroeck AH, et al. Assessment of Endothelial Dysfunction in Childhood Obesity and Clinical Use. Oxid Med Cell Longev. 2013;2013(2):1-19. doi: https://doi.org/10.1155/2013/174782

32. Лындина МЛ., Шишкин А.Н. Клинические особенности эндотелиальной дисфункции при ожирении и роль фактора курения // Регионарное кровообращение и микроциркуляция. 2018. - T. 17. - №2 - C. 18-25 Lyndina ML, Shishkin AN. Clinical features of endothelial dysfunctions in patients with obesity and role of smoking Regional blood circulation and microcirculation. 2018;17(2):18-25. doi: https://doi.org/10.24884/1682-6655-2018-17-2-18-25 


\section{ИНФОРМАЦИЯ ОБ АВТОРАХ [AUTHORS INFO]:}

*Авдеева Марина Владимировна, д.м.н., професcop [Marina V. Avdeeva, MD, PhD, professor]; адрес: Россия, 194100, Санкт-Петербург, ул. Литовская, д. 2 [address: 2 Litovskaya street, 194100 St. Petersburg, Russia];

ORCID: https://orcid.org/0000-0002-4334-5434; eLibrary SPIN: 6673-7195; e-mail: lensk69@mail.ru

Церцвадзе Лана Кахаберовна, аспирант [Lana K. Tsertsvadze, MD, postgraduate student]; ORCID: https://orcid.org/0000-0002-1304-0074; eLibrary SPIN: 8302-1703; e-mail: doct.lana@mail.ru

Щеглова Лариса Васильевна, д.М.Н., профессор [Larisa V. Scheglova, MD, PhD, professor];

ORCID: https://orcid.org/0000-0002-1059-4610; eLibrary SPIN: 6983-3999; e-mail: shcheglovalar@mail.ru

Василенко Владимир Станиславович, д.м.н., профессор [Vladimir S. Vasilenko, MD, PhD, professor];

ORCID: https://orcid.org/0000-0002-8833-304X; eLibrary SPIN: 1355-1790; e-mail: vasilenkovladi@yandex.ru

*Автор, ответственный за переписку / Corresponding author.

\section{ЦИТИРОВАТЬ:}

Церцвадзе Л.К., Авдеева М.В., Щеглова Л.В., Василенко В.С. Маркеры эндотелиальной дисфункции у пациентов юношеского и молодого возраста с гипоталамическим синдромом // Ожирение и метаболизм. - 2020. - Т. 17. - №3. С. 257-268. doi: https://doi.org/10.14341/omet12354

\section{TO CITE THIS ARTICLE:}

Tsertsvadze LK, Avdeeva MV, Scheglova LV, Vasilenko VS. Markers of endothelial dysfunction in adolescent and young patients with hypothalamic syndrome. Obesity and metabolism. 2020;17(3):257-268. doi: https://doi.org/10.14341/omet12354 